\title{
Desvalorização de ganhos e perdas monetárias em fumantes, fumantes em tratamento e não fumantes
}

\author{
Arianne de Sá Barbosa \\ Lisiane Bizarro \\ Universidade Federal do Rio Grande do Sul
}

\begin{abstract}
Resumo
Fumantes tendem a escolher recompensas imediatas de menor valor e desvalorizar as atrasadas de maior valor em modelos de desvalorização pelo atraso (DA). Entretanto, pouco se sabe sobre a escolha temporal de consequências apetitivas e aversivas de fumantes que decidem parar de fumar. Por isso, investigou-se a DA em uma tarefa com perdas e ganhos de valores monetários hipotéticos em fumantes $(n=20)$, fumantes em tratamento $(n=20)$ e não fumantes $(n=20)$, que também responderam o questionário de tolerância de Fagerström e o ASSIST. A DA foi maior para fumantes do que para não fumantes para ganhos (mas não perdas). Fumantes em tratamento não diferiram dos outros grupos, apresentando uma DA intermediária. Estes podem ser um subgrupo de fumantes menos impulsivos ou a decisão de parar de fumar reduziu a DA. A DA pode ser uma preditora do sucesso da cessação do tabagismo.
\end{abstract}

Palavras-chave: tabagismo; tomada de decisão; fumantes.

\begin{abstract}
Discounting of monetary gains and losses by smokers, smokers seeking treatment and non-smokers. Smokers tend to choose immediate reward of small magnitude and discount delayed reward of larger magnitude in Delay Discounting Models (DD). However, little is known about temporal choice of appetitive and aversive consequences among smokers who seek to quit smoking. DD was examined in a task of hypothetical monetary gains and losses in smokers $(n=20)$, smokers seeking treatment $(n=20)$ and non-smokers, who also answered the Fagerström Tolerance Questionnaire, and ASSIST. Smokers showed a greater DD than non-smokers in the appetitive condition (but not aversive). DD in smokers on treatment to quit was not different from remaining groups, and showed an intermediate DD. These smokers seeking treatment might either be a less impulsive subgroup of smokers or the decision to quit smoking reduced DD. DD might be an outcome predictor in smoking cessation treatment.
\end{abstract}

Keywords: smoking; decision-making; smokers.

$\mathrm{D}$ ecisões importantes no cotidiano dependem da escolha entre estímulos (reforçadores e punitivos) imediatos e atrasados (Catania, 1999; Critchfield \& Kollings, 2001; Deluty, 1978; Mazur,1996, 1998; Nevin, 1991; Rachlin, 1976). Diversos problemas comportamentais, geralmente englobam escolhas impulsivas (Moreira, 2007), como o autismo, o transtorno de déficit de atenção, a hiperatividade, a agressividade, a obesidade, a delinquência, o sedentarismo e a dependência química de substâncias (Hanna \& Ribeiro, 2005).

Iniciar, manter e parar o uso de substâncias psicoativas (SPAs) são comportamentos que exigem uma tomada de decisão. Indivíduos dependentes químicos desvalorizam as consequências atrasadas de seus atos mais do que indivíduos não-dependentes. Eles escolhem, por exemplo, a alternativa reforçadora imediata de sentir os efeitos apetitivos da SPA (usando-a) em detrimento de alternativas reforçadoras atrasadas, mesmo que de maior valor (por exemplo, melhor saúde, relacionamentos, emprego) (Johnson, Bickel, \& Baker, 2007). Isso sugere que a desvalorização pelo atraso (ou temporal) das consequências do comportamento é uma característica da dependência química (Bickel \& Johnson, 2003).

Um fumante, por exemplo, tenderia a escolher os efeitos reforçadores imediatos do fumar do que os efeitos reforçadores atrasados do não fumar (Bickel \& Marsch, 2001; Critchfield \& Kollings, 2001; Epstein et al., 2003). Fumantes tendem a desvalorizar mais quantias monetárias do que não fumantes (Bickel, Odum, \& Madden, 1999; Mitchell, 1999; Billieux, Van der Linden, \& Ceschi, 2007).

A desvalorização pelo atraso de ganhos monetários hipotéticos em fumantes não parece ser diferente daquela de dependentes de outras SPAs. Tanto fumantes quanto indivíduos dependentes de outras SPAs desvalorizaram mais quantias 
monetárias hipotéticas atrasadas do que indivíduos sem história de uso de cigarro e/ou dependência de outras substâncias (Businelle, McVay, Kendzor, \& Copeland, 2010). A taxa de desvalorização envolvendo recompensas financeiras foi superior em fumantes do que em nunca fumantes (Baker, Johnson, \& Bickel, 2003), mas fumantes leves não diferiram dos fumantes pesados (Johnson et al., 2007).

A redução no hábito de fumar pode levar à redução na desvalorização pelo atraso. Quando fumantes de nível de uso de cigarros moderado a pesado foram alocados randomicamente para uma condição de incentivo à redução do fumar por gerenciamento de contingências ou para um grupo sem este incentivo, observou-se que a desvalorização pelo atraso diminuiu apenas nos fumantes incentivados a reduzir o fumar (Yi et al., 2008). Portanto, o aumento da desvalorização em fumantes habituais pode ser um efeito reversível da dependência nicotínica. Também, neste sentido, sabe-se que uma abstinência de 24 horas resulta em uma maior desvalorização pelo atraso dos participantes em situações de escolha entre cigarro (imediata) ou dinheiro (atrasada) em relação a fumantes não abstinentes, mas não entre dinheiro (em menor quantidade e imediato) e dinheiro (em maior quantidade e atrasado) (Mitchell, 2004). Já a abstinência prolongada tende a diminuir a desvalorização pelo atraso (Allen, Moeller, Rhoades, \& Cherek, 1998; BrettevilleJensen, 1999; Kirby \& Petry, 2004; Petry, 2001b; Yi et al., 2008). Em geral, os estudos que avaliaram a desvalorização pelo atraso de indivíduos dependentes químicos utilizam situações apetitivas, ou seja, que envolvem a decisão entre dois estímulos reforçadores, em geral quantias monetárias hipotéticas (Johnson et al., 2007; Yoon et al., 2007; Epstein et al., 2003).

Para ampliar o entendimento da desvalorização pelo atraso em indivíduos fumantes, este estudo teve como objetivo investigar este fenômeno em situações apetitivas e em situações aversivas em fumantes, fumantes em tratamento e não fumantes. Investigou-se também a influência do nível de dependência do tabaco sobre a desvalorização pelo atraso, além da influência de variáveis sócio-demográficas como sexo e escolaridade.

\section{Metodologia}

\section{Participantes}

Participaram do estudo 62 indivíduos da região metropolitana de Porto Alegre, de ambos os sexos. Inicialmente, os participantes foram recrutados em um Centro de Atenção Psicossocial - Álcool e Drogas (CAPS-AD) de Gravataí (RS), onde foram convidados a participar da pesquisa. O convite inicial foi realizado através da divulgação da pesquisa entre os profissionais do serviço, que indicaram pacientes em tratamento no CAPS-AD. Além disso, flyers e cartazes foram distribuídos no centro e os sujeitos sensibilizados através deste material também foram convidados a participar do estudo.

$\mathrm{Na}$ instituição, são realizados regularmente grupos de cessação do tabagismo com 15 pacientes. Os grupos seguem as recomendações do Instituto Nacional do Câncer e têm como objetivo realizar o tratamento da dependência do cigarro. Esses grupos são constituídos por quatro sessões fechadas, estruturadas, semanais (grupo de tratamento $/ 1^{\circ}$ mês) e sessões abertas, não estruturadas (grupo de manutenção): duas quinzenais ( $2^{\underline{0}}$ mês) e 10 mensais (do $3^{\circ}$ ao $12^{-}$mês). As sessões abertas contam com a participação dos membros dos vários grupos fechados e são de manutenção do tratamento. Os demais participantes (fumantes e não fumantes) foram recrutados na mesma comunidade, através da técnica de bola de neve (Biernacki \& Waldorf, 1981). Ou seja, os primeiros participantes indicaram outros. Não houve pareamento dos grupos estudados, mas buscou-se uma equivalência de homens e mulheres.

Foram critérios de inclusão da amostra que os indivíduos tivessem, no mínimo, 18 anos e o ensino médio incompleto, e não fossem dependentes químicos de outra substância, além do tabaco. Os participantes poderiam estar usando medicações prescritas para o tratamento do tabagismo (bupropriona de $150 \mathrm{mg}$ e adesivo de nicotina de 7 , de 14 ou de $21 \mathrm{mg}$ e/ou goma de mascar de nicotina de $2 \mathrm{mg}$ ) ou prescritas para o tratamento de alguma outra doença. Dois participantes não preencheram os critérios de inclusão e não foram incluídos na amostra analisada. Portanto, os 60 participantes restantes (37 mulheres) pertenciam a um de três grupos delineados neste trabalho, cada um com 20 participantes: 1) grupo de fumantes, definidos como aqueles que declararam consumir pelo menos um cigarro por dia por, no mínimo, um ano (conforme Lopes, 2009; Rondina, Gorayeb, Botelho, \& Silva, 2005a; Rondina, Gorayeb, Botelho, \& Silva, 2005 b) e que não estavam em busca de tratamento para parar de fumar; 2) grupo de fumantes em tratamento para parar de fumar $(n=20)$ (definidos como aqueles fumantes que buscaram tratamento no CAPS-AD) e que estavam realizando as avaliações iniciais ou que haviam comparecido a uma ou mais sessões do grupo de tratamento ou do grupo de manutenção; 3) grupo de não fumantes autodeclarados $(n=20)$, (conforme Rondina et al., 2005a).

Todos os participantes assinaram um termo, consentindo de forma livre e esclarecida a sua participação no estudo. $\mathrm{O}$ projeto deste (protocolo de pesquisa $\mathrm{n}^{\mathbf{0}}$ 2009025) foi submetido e aprovado pelo Comitê de Ética do Instituto de Psicologia da UFRGS (registro $\mathrm{n}^{\mathrm{o}}$ 25000.089325/2006-58).

\section{Instrumentos}

Entrevista de dados sociodemográficos. Investigou dados sociais e demográficos dos participantes, tais como sexo, idade, renda e escolaridade máxima. O roteiro da entrevista era padronizado. Englobou o Critério de Classificação Econômica Brasil, que estima o poder de compra das pessoas e famílias urbanas, abandonando a pretensão de classificar a população em termos de "classes sociais". A divisão de mercado é de classes econômicas (Associação Brasileira de Empresas de Pesquisa, 2008a, 2008b).

Questionário de Tolerância de Fagerström (Heatherton, Kozlowski, Frecker, \& Fagerström, 1991). Este questionário consta de seis suposições maiores, hipotéticas, com as quais estaria relacionada a dependência nicotínica e que seriam o reflexo fiel do comportamento frente ao fumo, independentemente das interpretações pessoais, sendo as perguntas 1 e 4 as mais importantes para detecção do grau de dependência de nicotina (Halty, Huttener, Netto, Santos, \& Martins, 2002; Heatherton et 
al.,1991). Os escores permitem a classificação da dependência à nicotina em cinco níveis: muito baixo ( 0 a 2 pontos); baixo ( 3 a 4 pontos); moderado (5 pontos); alto (6 a 7 pontos); e muito alto (8 a 10 pontos). A consistência interna do Questionário é moderada, com um coeficiente alfa de Cronbach de 0,55 a 0,74 (Meneses-Gaya, Zuardi, Loureiro, \& Crippa, 2009).

Alcohol Smoking and Substance Involvement Screening Test (ASSIST 2.0). Foi aplicada a versão brasileira validada deste instrumento, denominada teste de triagem do envolvimento com álcool, tabaco e outras substâncias (Henrique, Micheli, Lacerda, Lacerda, \& Formigoni, 2004). Ele tem oito questões fechadas sobre o uso de nove classes de SPAs (tabaco, álcool, maconha, cocaína, anfetaminas, inalantes, sedativos, alucinógenos e opiáceos). Cada resposta corresponde a um escore, que pode variar de 0 a 4 pontos. A soma total dos pontos de todas as questões por substância pode variar de 0 a 20 pontos. O uso ocasional de uma substância é indicado pela faixa de escore de 0 a 3 pontos, o uso abusivo corresponde à faixa de escore de 4 a 15 pontos e a dependência química é indicada pelo escore igual ou superior a 16 pontos. A dependência de outras SPAs identificada no ASSIST foi usada como critério de exclusão.

Tarefa Experimental de Desvalorização pelo Atraso (TEDA) (Gonçalves, 2005). A tarefa foi realizada em um computador portátil (tela de 14.1", teclado ABNTII e mouse óptico), onde foram apresentadas duas sequências de slides adaptadas ao programa Microsoft Power Point 1997 (Microsoft, EUA). Uma sequência era de alternativas apetitivas (receber uma quantia de dinheiro) e outra, de aversivas (pagar dinheiro), apresentadas em ordem alternada entre os participantes. Em cada slide os participantes deveriam escolher entre duas alternativas (uma quantia de dinheiro imediata ou outra atrasada, em reais- $\mathrm{R} \$$ ).

As alternativas foram apresentadas nos slides como dois retângulos azuis (sinalizando a situação apetitiva) ou vermelhos (sinalizando a situação aversiva), de aproximadamente $5,0 \mathrm{~cm}$ $x 7,5 \mathrm{~cm}$, um à esquerda e outro à direita do campo visual, equidistantes do centro da tela. Abaixo de cada retângulo havia um botão virtual de aproximadamente $1,3 \mathrm{~cm} \times 1,7 \mathrm{~cm}$. $\mathrm{O}$ retângulo da direita sempre apresentava a quantia fixa de $\mathrm{R} \$$ $1.000,00$ e um dos oito atrasos distintos ( 1 semana, 1 mês, 6 meses, 1 ano, 3 anos, 5 anos, 10 anos ou 25 anos). O retângulo da esquerda sempre apresentava a alternativa da quantia imediata, que variava de $R \$ 1,00$ a $R \$ 1.000,00$, seguido da palavra HOJE. Para cada atraso, as quantias imediatas eram apresentadas em blocos de 30 tentativas em ordem crescente (de R $\$ 1,00$ a R $\$$ $1.000,00$ ) ou decrescente (de $\mathrm{R} \$ 1.000,00$ a $\mathrm{R} \$ 1,00$ ), de forma balanceada entre os sujeitos e os valores de atraso. Os valores de atraso eram sempre apresentados em ordem crescente (de 1 semana a 25 anos). Acima dos dois retângulos havia outro retângulo com uma pergunta que sinalizava ao participante em que situação estava (apetitiva ou aversiva). Para a situação apetitiva, a pergunta era: "Como você prefere receber?". Para a situação aversiva, a pergunta era: "Como você prefere pagar?". $\mathrm{O}$ fundo da tela em ambas as situações (apetitiva ou aversiva) tinha coloração cinza. A resposta consistiu em o participante posicionar o cursor em cima do botão virtual que representava sua escolha e apertar o botão esquerdo do mouse. Tal resposta provocava a mudança para uma tela escura (de coloração preta) e, após um intervalo de 1s, era apresentado um novo par de alternativas. As respostas dos participantes foram registradas pela pesquisadora em folhas de resposta.

\section{Procedimentos}

A coleta de dados dos participantes foi individual e nas dependências do CAPS-AD, em uma sala propícia para a execução do experimento: ambiente silencioso e isolado. Uma pesquisadora (ASB) realizou a coleta de dados de todos os participantes.

Após o consentimento do participante através do Termo de Consentimento Livre e Esclarecido, foi aplicada a ficha de dados sociodemográficos. A ordem de aplicação dos demais instrumentos foi randomizada. Antes do início da tarefa propriamente dita, os participantes da pesquisa fizeram uma sessão de treino, que consistia na exibição a quatro lâminas com alternativas de escolha que continham situações apetitivas e aversivas. Este treino era parte da padronização original da tarefa e tinha como objetivo habituar os participantes à tarefa e esclarecer dúvidas. A tarefa era executada em cerca de 30 minutos. O tempo total que os participantes despenderam para concluir os procedimentos relativos à aplicação dos instrumentos de pesquisa foi de aproximadamente uma hora. Apenas fumantes e fumantes em tratamento responderam ao Questionário de Tolerância de Fagerström.

\section{Análise dos dados}

A principal variável dependente foi o ponto de indiferença (PI) entre magnitudes imediatas e atrasadas medidas em Reais (R\$), que indica o ponto no qual o sujeito não apresentou preferência nem pela quantia imediata nem pela atrasada. $\mathrm{O}$ ponto de indiferença é a média entre a última quantia, anterior à mudança da escolha do participante, e a primeira quantia, logo após a mudança, seja da escolha da quantia imediata para a quantia atrasada ou da quantia atrasada para a imediata. Para cada participante, portanto, foram obtidos 16 pontos de indiferença (oito para a situação apetitiva e oito para a situação aversiva) (Coelho, Hanna, \& Todorov, 2003; Gonçalves, 2005).

Foi utilizada a análise da área sob a curva (Myerson, Green, \& Warusawitharana, 2001; Gonçalves, 2005). Neste tipo de análise, é levada em consideração a área formada pelos pontos de indiferença obtidos, sendo o resultado independente da equação que possa descrever a curva.

Os pontos de indiferença são apresentados como valor relativo à maior quantia atrasada $(\mathrm{R} \$ 1.000,00) \mathrm{e}$ o atraso como relativo ao maior atraso ( 25 anos), sendo transformados em pontos de indiferença relativos (PIR). Essa transformação permite que a área total possa variar de um valor mínimo de 0 (desvalorização completa) a um valor máximo de 1 (ausência de desvalorização). Desta forma, é possível calcular a área determinada por cada valor de atraso, assim como sua área total (Gonçalves, 2005). Sabendo-se que a área total determinada deveria ter um valor máximo de 1 e um valor mínimo de 0 , a área total determinada no gráfico, bem como a área de cada um dos sete trapézios determinados, foi calculada. Foram, então, calculadas as médias das áreas para cada uma das situações.

Com a constatação de que não havia homogeneidade e 
normalidade nos dados, foi utilizado o teste não-paramétrico $U$ de Mann-Whitney (Sheskin, 2000) para a comparação das médias das áreas totais e dos segmentos determinados por cada valor de atraso para comparação dos grupos (fumantes, fumantes em tratamento e não fumantes), das situações (apetitiva e aversiva), e de grupos definidos conforme variáveis sociodemográficas (sexo, renda, escolaridade) e, entre os fumantes e fumantes em tratamento, o nível de dependência. Foram realizadas comparações em pares de grupos, usando os não fumantes como grupo de referência, o que torna o teste $U$ de Mann Whitney indicado. Para as comparações dos três grupos simultaneamente (fumantes, fumantes em tratamento e não fumantes) foi feita a correção de Bonferroni, que torna o teste mais conservador do que a análise de Kruskall-Wallis.

A análise dos dados foi feita com os pacotes estatísticos Statistical Package for Social Sciences (SPSS), versão 16.0, $R$ versão 2.10.0 (R Development Core Team, 2009) e com a planilha eletrônica Microsoft Excel 2007. Todos os testes foram realizados assumindo um nível de significância $\alpha=5 \%$.

\section{Resultados}

Os fumantes tinham em média 36 anos $(D P=10)$, começaram a fumar com 15,5 anos $(D P=1,02)$ e tinham nível de dependência de nicotina de baixo a médio $(4,4$ pontos no

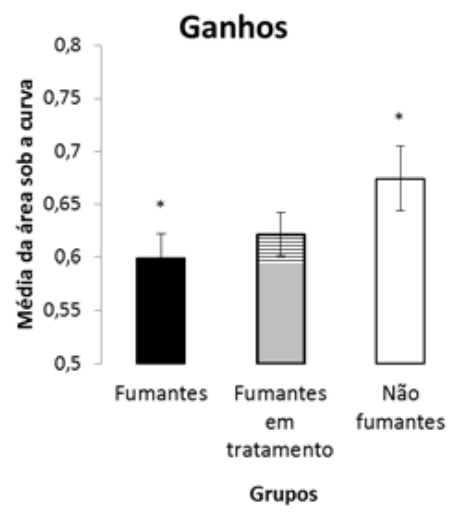

Questionário de Tolerância de Fagerström). Os fumantes em tratamento tinham em média 42 anos $(D P=9)$, começaram a fumar em média com 15 anos $(D P=0,94)$ e tinham nível de dependência de nicotina de médio a elevado (5,3 pontos no Questionário de Tolerância de Fagerström). Os não fumantes tinham em média 35 anos $(D P=12)$ e não responderam ao questionário que indicava nível de dependência de nicotina.

A desvalorização pelo atraso de todos os participantes foi semelhante na situação apetitiva $(M=0,63 ; D P=0,12)$ e aversiva $(M=0,64 ; D P=0,12)(U=1849.0 ; p=0,8)$. Mas, apesar deste resultado geral, fumantes escolheram de forma diferente dos não fumantes apenas na situação apetitiva. Nesta situação, os fumantes exibiram maior desvalorização pelo atraso do que não fumantes na análise de área sob a curva total $(U=123 ; p=0,04)$ e em diversos atrasos analisados separadamente. Já o grupo de fumantes em tratamento não foi diferente dos fumantes $(U=157$; $p=0,25)$ ou dos não fumantes $(U=150 ; p=0,18)$, com exceção de um atraso. A média da área sob a curva do grupo de fumantes em tratamento $(M=0,62 ; D P=0,09)$ foi intermediária entre os grupos de fumantes $(M=0,60 ; D P=0,11)$ e não fumantes $(M=$ $0,67 ; D P=0,14)$, na situação apetitiva. Já na situação aversiva, fumantes $(M=0,6411 ; D P=0,1421)$, fumantes em tratamento $(M=0,6052 ; D P=0,0881)$ e não fumantes $(M=0,6620 ; D P=$ $0,1275)$ tiveram médias muito semelhantes, de modo que não diferiram estatisticamente (Figura 1).

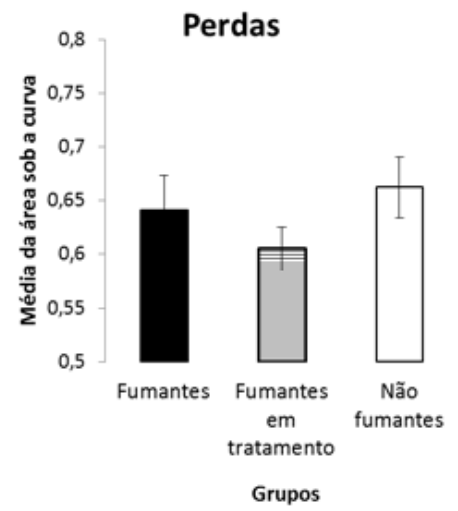

\section{Figura 1}

Comparação da média da área sob a curva de todos os atrasos para ganhos e perdas monetárias dos grupos de fumantes, fumantes em tratamento e não fumantes. Barras de erro indicam erro padrão da média. Asteriscos indicam os grupos que são diferentes entre si: fumantes apresentaram uma área sob a curva menor, portanto, desvalorizam mais ganhos monetários atrasados do que fumantes, mas esta diferença não foi observada para perdas monetárias atrasadas. Os fumantes em tratamento não diferem dos demais grupos.

Para avaliar a influência da severidade da dependência do tabaco na desvalorização pelo atraso, os grupos de fumantes e de fumantes em tratamento foram classificados quanto ao seu nível de dependência a partir do Questionário de Tolerância de Fagerström (QTF). Foram encontrados 17 participantes de nível baixo de dependência (sendo 10 fumantes e 07 fumantes em tratamento), 04 participantes de nível médio de dependência (sendo 01 fumante e 03 fumantes em tratamento) e 19 participantes de nível alto de dependência (sendo 09 fumantes e 10 fumantes em tratamento). Os grupos não diferiram na desvalorização pelo atraso nas situações apetitiva e aversiva.

Da mesma forma, as variáveis sociodemográficas sexo, idade e escolaridade não influenciaram a desvalorização pelo atraso dos participantes. Apenas as mulheres, na situação apetitiva, parecem desvalorizar mais se forem fumantes em comparação com as não fumantes $(U=40 ; p=0,04)$.

Em relação à renda, não houve diferença significativa em relação à tomada de decisão dos grupos analisados na situação 
aversiva. Mas, na situação apetitiva, foi encontrada uma desvalorização menor nos participantes com renda familiar acima de $\mathrm{R} \$ 3.944,00(n=13)$ quando comparados com os participantes com renda abaixo de $\mathrm{R} \$ 861,00(n=11 ; U=30 ; p=0,02)$ ou abaixo de $\mathrm{R} \$ 2.256,00(n=36 ; U=133 ; p=0,02)$.

\section{Discussão}

A semelhança entre a desvalorização pelo atraso para a situação apetitiva e a aversiva foi diferente do encontrado por Gonçalves (2005), que verificou uma desvalorização maior em situações apetitivas do que em aversivas nesta mesma tarefa entre universitários. Diversos fatores podem ter contribuído para esta diferença, entre eles o nível socioeconômico e educacional da amostra do presente estudo, que podem afetar a desvalorização pelo atraso (De Wit, Flory, Acheson, McCloskey, \& Manuk, 2007).

$\mathrm{O}$ achado de que fumantes desvalorizam mais recompensas atrasadas do que não fumantes não é novo, pois muitos estudos já compararam estes grupos em situações de escolha entre dois estímulos apetitivos (Baker et al., 2003; Bickel et al., 1999; Bickel \& Marsch, 2001; Bickel \& Johnson, 2003; Billieux et al., 2007; Burt, Dinh, Peterson, \& Sarason, 2000; Critchfield \& Kollings, 2001; Epstein et al., 2003; Eysenck, Tarrant, \& Woolf, 1960; Johnson et al., 2007; Jorm, Rodgers, Christensen, Henderson, \& Korten, 1999; Kirby, Petry, \& Bickel, 1999; Mitchel, 1999). A semelhança entre indivíduos com diferentes níveis de dependência também concorda com os resultados de Johnson e colaboradores (2007). Mas, o achado de que o grupo de fumantes em tratamento não foi diferente dos demais pode deverse à composição heterogênea deste grupo, que tem indivíduos que não deixaram de fumar e indivíduos que continuam fumando, sendo que todos têm em comum o fato de terem procurado tratamento. Por outro lado, pode indicar que este é um grupo com uma desvalorização de ganhos atrasados intermediária entre os grupos de fumantes e não fumantes. Este resultado é inédito na área e pode contribuir para o desenvolvimento de melhores estratégias terapêuticas.

Em um primeiro momento, pode-se considerar que a abstinência ou a redução da frequência do comportamento de fumar poderia ter diminuído a desvalorização pelo atraso dos fumantes em tratamento (Bickel et al., 1999; Mitchell, 2004, Yi et al., 2008). Tendo em vista que os fumantes em tratamento do presente estudo não desvalorizaram mais do que os fumantes ou tiveram uma área sob a curva maior, indicando uma tendência a desvalorizar menos na situação apetitiva, o efeito da abstinência prolongada poderia ser responsável por esta tendência. Mas, esta explicação não é satisfatória, visto que todos os participantes do grupo de fumantes em tratamento estão buscando ou mantendo uma abstinência há pouco tempo (alguns nem pararam de fumar completamente).

Então, o fato de o grupo de fumantes em tratamento ser um grupo intermediário em relação à desvalorização pelo atraso em situações apetitivas pode indicar que se trata de um subgrupo de fumantes que, por desvalorizar menos, procura tratamento. $\mathrm{Ou}$, alternativamente, pode ser formado por um grupo de fumantes que mudou a estratégia de escolha, desvalorizando menos após procurar tratamento ou ser incentivado a mudar o comportamento de fumar (Yi et al., 2008).

Sabe-se que, quando avaliados por uma escala, ex-fumantes são menos impulsivos que fumantes (Rondina et al., 2005a). Questiona-se, porém, se fumantes em tratamento (alguns dos quais virão a ser ex-fumantes) já seriam, em princípio, menos impulsivos em tarefas de desvalorização pelo atraso do que fumantes que não buscam tratamento. A hipótese do tratamento do tabagismo interferir na desvalorização também é válida (Yi et al., 2008), mas, se muitos dos participantes do grupo de fumantes em tratamento ainda estavam no início deste, é questionável se a motivação inicial do grupo de fumantes em tratamento não interferiu mais na sua desvalorização pelo atraso do que o tratamento propriamente dito. Desta forma, também é possível que a desvalorização pelo atraso possa ser usada como uma medida de motivação para o tratamento, apesar de um estudo usando outro modelo de tomada de decisão não ter encontrado evidência desta relação (Harmsen, Bischof, Brooks, Hohagen, \& Rumpf, 2006).

Poderia ser argumentado que, por serem mais velhos, a idade dos participantes do grupo de fumantes em tratamento poderia ter interferido na desvalorização pelo atraso. A média de idade dos participantes do grupo de fumantes em tratamento foi de 42 anos, enquanto a do grupo de fumantes e não fumantes foram 36 e 35 anos, respectivamente. A taxa de desvalorização pelo atraso é maior em adultos jovens, declinando na faixa etária de 30 anos e se mantendo relativamente constante após esta fase (Green, Fry, \& Myerson, 1994; Green, Myerson, Lichtman, Rosen, \& Fry, 1996; Reimers, Maylor, Stewart, \& Chater, 2009). A média das idades dos grupos fica acima dos 30 anos e, quando os participantes foram divididos em grupos etários e estes foram comparados, não foi encontrada diferença na desvalorização pelo atraso nesta amostra. Fumantes com mais de 40 anos podem apresentar maior probabilidade de tentar a abstinência do que aqueles mais jovens, devido à maior incidência de doenças de tabaco relacionadas com o avançar da idade (Breslau \& Peterson, 1996), mas idade, sexo e escolaridade não parecem ter influenciado a desvalorização pelo atraso dos participantes do presente estudo. Já as diferenças de desvalorização pelo atraso devido à renda familiar foram consistentes com outros estudos (De Wit et al., 2007; Green et al., 1996; Harrison, Lau, \& Williams, 2002).

Na situação aversiva, os grupos não diferiram. Diante deste resultado, pode-se supor que fumantes não seriam afetados de maneira diferente por contingências aversivas do que são os não-fumantes e os fumantes em tratamento. Logo, é possível que o controle aversivo não seja efetivo sobre o comportamento de fumar. Assim, o uso de controle aversivo no tratamento do tabagismo parece ser uma estratégia menos eficiente do que o controle apetitivo, como aquele usado por Yi et al. (2008) para incentivar a redução do consumo de cigarros.

Este estudo, portanto, ampliou o entendimento sobre a tomada de decisão de indivíduos fumantes e fumantes em tratamento, através da investigação sobre a desvalorização pelo atraso, em situações apetitivas e em situações aversivas. Estas informações sugerem que um tratamento que utilize controle apetitivo com consequências imediatas para o comportamento 
de evitar o fumar pode ser mais efetivo do que consequências atrasadas ou controle aversivo. A hipótese de que a desvalorização pelo atraso possa ser um indicador implícito de motivação para a mudança poderá ser testada em futuros estudos. Estratégias de intervenção e tratamento mais eficazes para o tabagismo podem ter a desvalorização pelo atraso (o continuum autocontroleimpulsividade) como meio de interferir nas escolhas de iniciar a fumar, manter o uso e parar de fumar.

\section{Referências}

Allen, T. J., Moeller, F. G., Rhoades, H. M., \& Cherek, D. R. (1998). Impulsivity and history of drug dependence. Drug and Alcohol Dependence, 50, 137-145.

Associação Brasileira de Empresas de Pesquisa (2008a). Adoção do CCEB 2008: critério de classificação econômica Brasil. Recuperado de http://www.abep. org/?usaritem=arquivos\&iditem $=23$

Associação Brasileira de Empresas de Pesquisa (2008b). Critério de classificação econômica Brasil. Recuperado de http://www.abep.org/?usaritem=arquivo s\&iditem $=23$

Baker , F., Johnson , M. W., \& Bickel , W. K. (2003). Delay discounting in current and never-before cigarette smokers: similarities and differences across commodity, sign, and magnitude. Journal of Abnormal Psychology $112,382-392$

Bickel, W. K., \& Johnson, M. W. (2003). Delay discounting: a fundamental behavioral process of drug dependence. In G. Loe-wenstein, D. Read \& R. F. Baumeister (Orgs.), Time and Decision (pp. 419-440). Nova Iorque: Russel Sage Foundation.

Bickel, W. K., \& Marsch, L. A. (2001). Toward a behavioural economic understanding of drug dependence: delay discounting processes. Addiction, 96, 73-86.

Bickel, W. K., Odum, A. L., \& Madden, G. J. (1999). Impulsivity and cigarette smoking: delay discounting in current, never and ex-smokers. Psychopharmacology, 146, 447-454.

Biernacki, P., \& Waldorf, D. (1981). Snowball Sampling. Sociological Methods and Research, 5(2), 141-163.

Billieux, J., Van der Linden, M., \& Ceschi, G. (2007). Which dimensions of impulsivity are related to cigarette craving? Addictive Behaviors, 32, 1189-1199.

Breslau, N., \& Peterson, E. L. (1996). Smoking cessation in young adults: age at initiation of cigarette smoking and other suspected influences. American Journal of Public Health, 86, 214-220.

Bretteville -Jensen, A. L. (1999). Addiction and discounting. Journal of Health and Economics, 18, 393-407.

Burt, R. D., Dinh, K. T., Peterson, A. V., \& Sarason, I. G. (2000). Predicting adolescent smoking: a prospective study of personality variables. Preventive Medicine, 30, 115-25.

Businelle, M. S., McVay, M. A, Kendzor, D., \& Copeland, A. (2010). A comparison of delay discounting among smokers, substance abusers, and non-dependent controls. Drug and Alcohol Dependence, 112(3), 247-250.

Catania, A. C. (1999). Aprendizagem: comportamento, linguagem e cognição. Porto Alegre: Artmed.

Coelho, C., Hanna, E. S., \& Todorov, J. C. (2003). Magnitude, atraso e probabilidade de reforço em situações hipotéticas de risco. Psicologia: Teoria e Pesquisa, 19(3), 269-278.

Critchifield, T. S., \& Kollings, S. H. (2001). Temporal discounting: basic research and the analysis of socially important behavior. Journal of Applied Behavior Analysis, 34, 101-122.

Deluty, M. Z. (1978). Self-control and impulsiveness involving aversive events. Dissertation Abstracts International, 38(9-B), 4502
De Wit, H., Flory, J. D., Acheson, A., McCloskey, M., \& Manuck, S. B. (2007). IQ and nonplanning impulsivity are independently associated with delay discounting in middle-aged adults. Personality and Individual Differences, $42,111-121$

Epstein, L. H., Richards, J. B., Saad, F. G., Paluch, R. A., Roemmich, J. N., \& Lerman, C. (2003). Comparison between two measures of delay discouting in smokers. Experimental and clinical psychopharmacology, 11(2), 131-138.

Eysenck, H. J., Tarrant, M., \& Woolf, M. (1960). Smoking and personality. British Medical Journal, 1, 1456-60.

Gonçalves, F. L.(2005). Desvalorização pelo atraso em situações apetitivas e aversivas (Tese de doutorado). Universidade de São Paulo, São Paulo. Recuperado de <http://www.teses.usp.br/teses/disponiveis/47/47135/tde04072006-142906/>

Green, L., Fry, A. F., \& Myerson, J. (1994). Discounting of delayed rewards: a lifespan comparison. Psychological Science, 5, 33-36.

Green, L., Myerson, J., Lichtman, D., Rosen, S., \& Fry, A. (1996). Tempora discounting in choice between delayed rewards: the role of age and income. Psychology and Aging, 11, 79-84.

Halty, L. S., Huttener, M. D., Netto, I. C. O., Santos, V. A., \& Martins, G. (2002). Análise da utilização do Questionário de Tolerância de Fargestron (QTF) como medida da dependência de nicotina. Jornal Brasileiro de Pneumologia, $28,180-86$

Hanna, E. S., \& Ribeiro, M. R. (2005). Autocontrole: um caso especial de comportamento de escolha. In J. Abreu-Rodrigues \& M. R. Ribeiro (Orgs.), Análise do Comportamento: pesquisa, teoria e aplicação (pp. 175-186). Porto Alegre: Artmed.

Harmsen, H., Bischof, G., Brooks, A., Hohagen, F., Rumpf, H.-J. (2006). The relationship between impaired decision-making, sensation seeking and readiness to change in cigarette smokers. Addictive Behaviors, 31(4), 581-92.

Harrison, G. W., Lau, M. I., \& Williams, M. B. (2002). Estimating individual discount rates in Denmark: a field experiment. American Economic Review, 92, 1606-1617.

Heatherton, T. F., Kozlowski, L.T., Frecker, R. C., \& Fagerström, K. O. (1991). The Fagerström test for nicotine dependence: a revision of the Fagerström Tolerance Questionnaire. British Journal of Addiction, 86, 1119-27.

Henrique, I. F. S., Micheli, D., Lacerda, R. B., Lacerda, L. A., \& Formigoni, M. L. O. S (2004). Validação da versão brasileira do teste de triagem do envolvimento com álcool, cigarro e outras substâncias (Assist). Revista da Associação Médica Brasileira, 50(2), 199-206.

Johnson, M. W., Bickel, W. K., \& Baker, F. (2007). Moderate drug use and delay discounting: a comparison of heavy, light, and never smokers. Experimental and Clinical Psychopharmacology, 15(2), 187-194.

Jorm, A. F., Rodgers, B., Christensen, H., Henderson, S., \& Korten, A. E. (1999). Smoking and mental health: results from a community survey. Medical Journal of Australia, 170, 74-77.

Kirby, K. N., Petry, N. M., \& Bickel, W. K. (1999). Heroin addicts have highter discount rates for delayed rewards than non-drug-using controls. Journal of Experimental Psychology General, 128(1), 78-87.

Kirby, K. N., \& Petry, N. M. (2004). Heroin and cocaine abusers have higher discount rates for delayed rewards than alcoholics or non-drug-using controls. Addiction, 99, 461-471

Lopes, F. M. (2009). Viés Atencional em jovens fumantes (Dissertação de Mestrado). Universidade Federal do Rio Grande do Sul, Porto Alegre. (000711376)

Mazur, J. E. (1996). Procrastination by pigeons: preference for larger, more delayed work requirements. Journal of the Experimental Analysis of Behavior, 65(1), 159-171.

Mazur, J. E. (1998). Procrastination by pigeons with fixed-interval response requirements. Journal of the Experimental Analysis of Behavior, 69(2), 185-197

Meneses-Gaya, I. C., Zuardi, A. W., Loureiro, S. R., \& Crippa, J. A. S. (2009). 
As propriedades psicométricas do Teste de Fagerström para Dependência de Nicotina. Jornal Brasileiro de Pneumologia, 35(1), 73-82.

Mitchell, S. H. (1999). Measures of impulsivity in cigarette smokers and nonsmokers. Psychopharmacology, 146, 455-464.

Mitchell , S. H. (2004). Effects of short-term nicotine deprivation on decision making: delay, uncertainty, and effort discounting. Nicotine \& Tobacco Research, 6, 819-828.

Moreira, J. M. (2007). Efeitos da variação comportamental durante o atraso do reforço sobre a escolha por autocontrole (Dissertação de Mestrado). Universidade de Brasília, Brasília. Recuperado de http://hdl.handle. net/10482/2902

Myerson, J., Green, L., \& Warusawitharana, M. (2001). Area under the curve as a measure of discounting. Journal of the Experimental Analysis of Behavior, $76(2), 235-243$.

Nevin, J. A. (1991). Behavior analysis and global survival. In I. Waris (Org.), Human behaviour in today's world (pp. 39-49). Nova Iorque: Praeger.

Petry, N. M. (2001). Delay discounting of money and alcohol in actively using alcoholics, currently abstinent alcoholics, and controls. Psychopharmacology, 154, 243-250.

Rachlin, H. (1976). Behavior and Learning. São Francisco: Freeman.
Reimers, S., Maylor, E. A., Stewart, N., \& Chater, N. (2009). Associations between a one-shot delay discounting measure and age, income, education and real-world impulsive behaviour. Personality and Individual Differences, 47, 973-978.

Rondina, R. C., Gorayeb, R., Botelho, C., \& Silva, A. M. C. (2005a). Estudo comparativo entre características de personalidade de universitários fumantes, ex-fumantes e não-fumantes. Revista de Psiquiatria do Rio Grande do Sul, $27,140-150$

Rondina, R. C., Gorayeb, R., Botelho, C. , \& Silva, A. M. C. (2005b). A relação entre tabagismo e características sócio-demográficas em universitários. Psicologia, Saúde \& Doenças, 6, 35-45.

Sheskin, D. J. (2000). Handbook of Parametric and Nonparametric Statistical Procedures. Nova Iorque: Chapman \& Hall.

Yi, R., Johnson, M. W., Giordano, L. A., Landes, R. D., Badger, G. J., \& Bickel, W. K. (2008). The effects of reduced cigarette smoking on discounting future rewards: an initial evaluation. The Psychological Record, 58, 163-174.

Yoon, J. H., Higgins, S. T., Heil, S. H., Sugarbaker, R. J., Thomas, C. S., \& Badger, G. J. (2007). Delay discounting predicts postpartum relapse to cigarette smoking among pregnant women. Experimental and clinical psychopharmacology, 15(2), 176-186.

Arianne de Sá Barbosa, mestre em Psicologia pela Universidade Federal do Rio Grande do Sul, é Psicóloga do Centro de Atenção Psicossocial Álcool e Drogas (CAPS-AD) da Prefeitura Municipal de Gravataí-RS, é Psicóloga Clínica e Organizacional da Clínica Conviver Psicologia. Membro do Laboratório de Psicologia Experimental, Neurociências e Comportamento (LPNeC-UFRGS), é representante do Conselho Regional de Psicologia no Conselho Municipal de Saúde de Gravataí. Endereço para correspondência: Universidade Federal do Rio Grande do Sul, Instituto de Psicologia, Departamento de Psicologia do Desenvolvimento e da Personalidade. Rua Ramiro Barcelos 2600, sala 105. Santana. CEP 90035-003. Porto Alegre, RS. Email: ariannedesa@yahoo.com.br

Lisiane Bizarro, doutora em Psicologia pelo Institute Of Psychiatry King's College London e PhD em Psicologia pelo Institute of Psychiatry King's College London, é professora associada do departamento de Psicologia do Desenvolvimento e da Personalidade e professora permanente do Programa de pós-graduação em Psicologia da Universidade Federal do Rio Grande do Sul (UFRGS). Pesquisadora do Núcleo de Estudos Translacionais em Adições do Laboratório de Psicologia Experimental, Neurociências e Comportamento (LPNeC -UFRGS). Email: lisiane.bizarro@gmail.com ou lisiane.bizarro@ufrgs.br 